\title{
Letter to the Editors
}

\section{Ceftobiprole associated agranulocytosis after drug rash with eosinophilia and systemic symptoms induced by vancomycin and rifampicin}

\author{
Thomas Wendland, ${ }^{1 *}$ Barbara Daubner $^{2 *} \&$ Werner J. Pichler ${ }^{2,3}$ \\ ${ }^{1}$ University Clinic of Internal Medicine, Inselspital Bern, Switzerland, ${ }^{2} A D R-A C$ GmbH, Holligenstrasse 91, 3008 Bern, Switzerland \\ and ${ }^{3}$ University Clinic of Rheumatology, Clinical Immunology and Allergology, Inselspital Bern, Switzerland
}

Drug rash with eosinophilia and systemic symptoms (DRESS) is a severe drug hypersensitivity reaction [1]. Sulfonamides, antiepileptics, allopurinol, minocyclin, diltiazem, vancomycin and $\beta$-lactam antibiotics are the most common elicitors. Symptoms include fever, skin rash, facial oedema, organ involvement such as hepatitis or interstitial nephritis, pneumonitis and myocarditis. Lymphadenopathy and splenomegaly may occur. DRESS syndrome is characterized by the presence of eosinophilia and atypical lymyphocytes in blood. The syndrome occurs within 1-12 weeks after initiating drug treatment [2]. Mortality is around $10 \%$, most often due to liver failure. Drug-specific circulating T-cells seem to play a central role in its pathogenesis [3].

Ceftobiprole is a fifth-generation broad-spectrum cephalosporin with activity against many Gram-positive and Gram-negative bacteria. It is the first cephalosporin with activity against methicillin-resistant Staphylococcus aureus and coagulase-negative staphylococci. It is approved in Switzerland, Canada and the European Union under the trade name of Zeftera ${ }^{\circledR}$ for the treatment of complicated skin and skin-structure infections, including diabetic foot infection without osteomyelitis [4]. Most common side-effects are dysgeusia, nausea and headache. Hypersensitivity with rash and pruritus has been described in rare cases [5]. The manufacturer recommends caution with treatment longer than 14 days due to limited experience with the drug [6].

Agranulocytosis following treatment with cephalosporins is a rare but recognized side-effect [7]. It typically occurs after prolonged treatment. Here we present a first report on ceftobiprole-associated agranulocytosis.

A 65-year-old woman underwent elective cardiac surgery to replace the mitral valve due midgrade mitral valve insufficiency that had caused several episodes of leftsided heart failure over the past 6 years. The medical history showed recurrent cerebrovascular ischaemia 13

*Both authors contributed equally. and 7 years before admission and chronic renal insufficiency. Anticoagulation was done for 6 years with acetylsalicylic acid and was later changed to phenprocoumon. The patient was being treated with metoprolol $100 \mathrm{mg}$ once daily and perindopril $10 \mathrm{mg}$ once daily. A summary of clinical events, antibiotic treatment and laboratory results is given in Figure 1A. Mitral valve replacement was combined with bypass surgery due to right coronary artery stenosis. Following surgery, the patient exhibited a complete atrioventricular block, requiring pacemaker implantation. Twelve days after surgery, the patient developed fever. Laboratory tests showed a C-reactive protein of $222 \mathrm{mg} \mathrm{l}^{-1}$ and a leukocytosis $\left(14.3 \mathrm{gl}^{-1}\right)$. Two of four blood cultures and probes from the sternal wound and pus grew coagulase-negative staphylococci (resistant to penicillin, oxacillin and ciprofloxacin, sensitive to gentamicin, clindamycin, tetracyclin, vancomycin, sulfamethoxazole/ trimethoprim and rifampicin). Sternal osteomyelitis was diagnosed and endocarditis was suspected, first not documented, but later confirmed by echocardiography. Repeated blood cultures were negative. Treatment was initially begun with vancomycin $1 \mathrm{~g}$ I.V. twice daily, rifampicin $600 \mathrm{mg}$ P.O. once daily and gentamicin $1 \mathrm{mg} \mathrm{kg}^{-1}$ i.v. three times daily. Gentamicin and vancomycin were briefly replaced with sulfamethoxazole/trimethoprim $800 \mathrm{mg} /$ $160 \mathrm{mg}$ P.O. three times daily 16 days after diagnosis, but soon discontinued, and vancomycin and gentamicin were administered again, because of vegetations on the mitral valve.

Eleven days after modification of antibiotic therapy, the patient was feverish with an exanthema of the trunk, arms and legs. She was icteric. Laboratory tests showed a C-reactive protein of $104 \mathrm{mg} \mathrm{l}^{-1}$, an eosinophilia of $0.61 \mathrm{~g} \mathrm{l}^{-1}$, atypical lymphocytes $0.5 \%$, a bilirubinaemia of $50 \mu \mathrm{moll}^{-1}$, elevation in transaminases (alanine aminotransferase $218 \mathrm{UI}^{-1}$ and aspartate aminotransferase $758 \mathrm{Ul}^{-1}$ ), alkaline phosphatase was $450 \mathrm{Ul}^{-1}, \gamma$-glutamyltranspeptidase $980 \mathrm{UI}^{-1}$ and lactate 


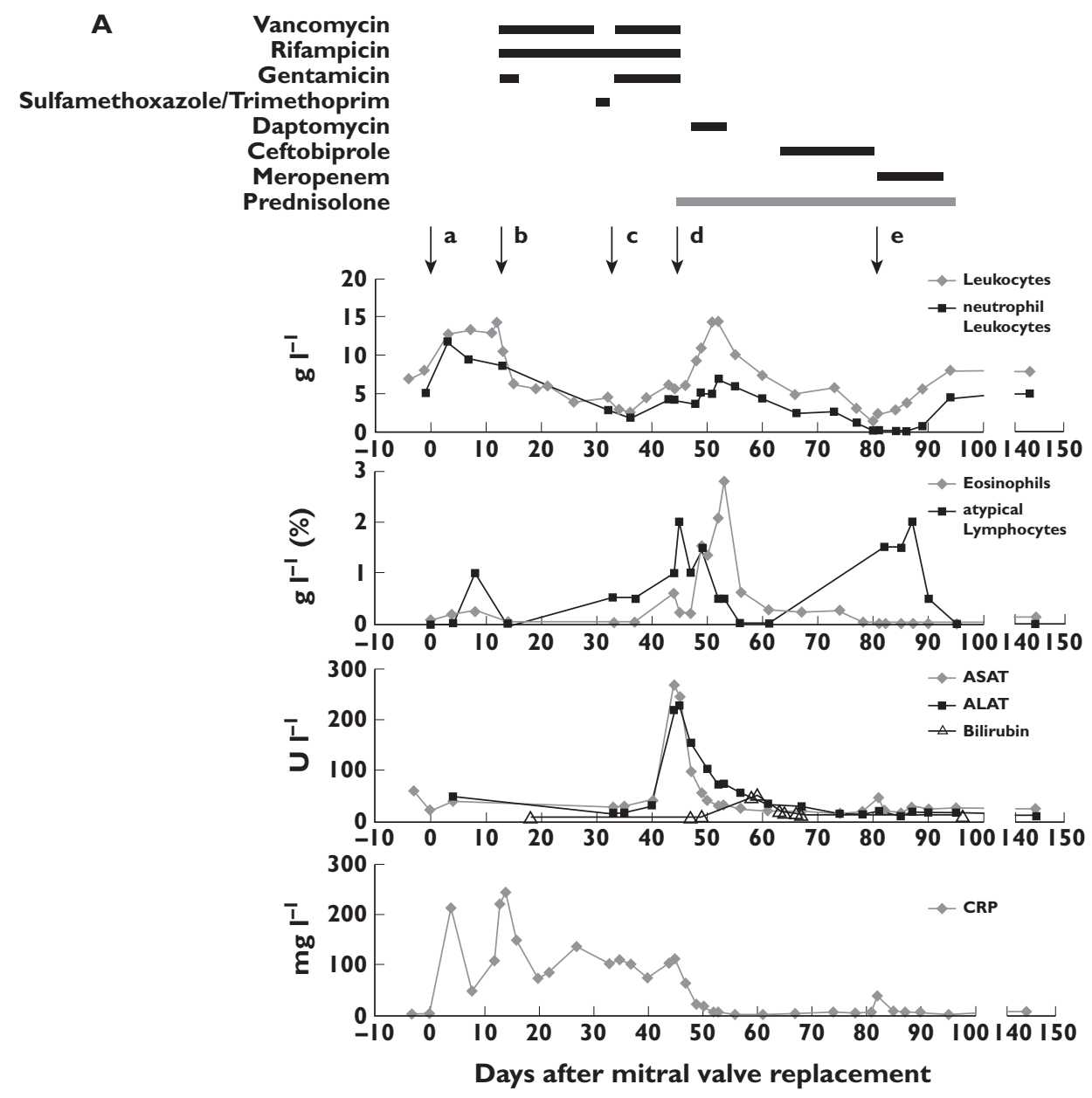

\section{B}

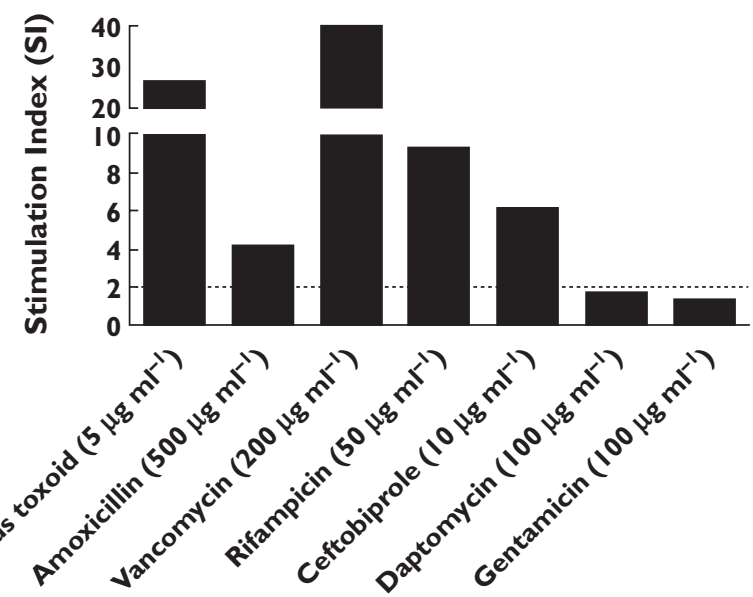

\section{Figure 1}

(A) Treatment history and laboratory data. The duration of antibiotic treatment is shown by the black bars and the duration of prednisolone treatment by the grey bar. Arrows denote the following: a, mitral valve replacement; b, sternal osteomyelitis; c, endocarditis; d, DRESS; and e, agranulocytosis. The number of leukocytes, eosinophil and neutrophil granulocytes are given in grams per litre, and atypical lymphocytes are given as the percentage of leukocytes. Abbreviations: ALAT, alanine aminotransferase $\left(\mathrm{U} \mathrm{I}^{-1}\right)$; ASAT, aspartate aminotransferase $\left(\mathrm{U} \mathrm{I}^{-1}\right)$, Bili, Bilirubin $\left(\mathrm{U} \mathrm{I}^{-1}\right)$; and $\mathrm{CRP}, \mathrm{C}^{-}$reactive protein $\left(\mathrm{mg} \mathrm{I}^{-1}\right)$. $(\mathrm{B})$ Proliferation after drug stimulation of peripheral blood mononuclear cells isolated from the patient. The lymphocyte transformation test (LTT) was performed as described before [8]. Stimulation indices (SIs) were calculated from triplicate cultures as counts per minutes in culture medium with antigen divided by counts per minutes in culture medium without antigen. As a positive control, cells of the patient were stimulated with tetanus toxoid. An $\mathrm{SI}_{\llcorner\mathrm{LT}}>2$ is considered as positive [8]. 
dehydrogenase $1110 \mathrm{Ul}^{-1}$. A DRESS syndrome was suspected, and treatment was discontinued for 2 days. Prednisolone $100 \mathrm{mg}$ once daily was given. Treatment was restarted with daptomycin $6 \mathrm{mg} \mathrm{kg}^{-1}$ I.V. once daily. After 7 days of daptomycin treatment, the number of eosinophils $\left(2.81 \mathrm{~g} \mathrm{l}^{-1}\right)$ increased. Antibiotic treatment was discontinued for 9 days, and eosinophil counts, bilirubin and transaminases normalized. Treatment with ceftobiprole $500 \mathrm{mg}$ I.V. three times daily was started, and predisolone was gradually reduced to $5 \mathrm{mg}$ once daily. Perindopril was reinstalled 5 days after start of ceftobiprole treatment, and acetylsalicylic acid $100 \mathrm{mg}$ once daily and metoprolol were given again 11 days after start of ceftobiprole treatment.

After 18 days of ceftobiprole treatment, the patient became progressively tired, and the laboratory showed a neutropenia of $0.08 \mathrm{gl}^{-1}$. The following day, neutrophils were down to zero and atypical lymphocytes were detected (1.5\%). Ceftobiprole and acetylsalicylic acid were stopped as being the most likely cause, and prednisolone was given at $60 \mathrm{mg}$ once daily. Since the patient had neutropenic fever that day without focus, empirical treatment with meropenem $1 \mathrm{~g}$ I.V. three times daily was begun. Neutrophil leukocytes recovered 9 days after discontinuation of ceftobiprole and acetylsalicylic acid to normal levels without the use of haematopoetic growth factors, and meropenem was stopped. The patient was discharged with a neutrophil count of $4.42 \mathrm{~g} \mathrm{l}^{-1}$. Acetylsalicylic acid was reinstalled and was tolerated well, as neutropenia did not recur in an 8 week follow-up. The incident was reported to the Swiss Pharmacovigilance Center.

Allergy testing revealed a positive skin test for amoxicillin, but negative results for vancomycin, rifampicin, daptomycin and a weak positive result for gentamicin. In vitro lymphocyte transformation testing, performed as described before [8], showed clear positive responses to amoxicillin, vancomycin, rifampicin and ceftobiprole but negative results for daptomycin and gentamicin (Figure 1B).

Drug-induced agranulocytosis is an idiosyncratic adverse reaction to drugs which may be life threatening [9]. It results in a severe reduction of granulocytes in the peripheral blood with an absolute neutrophil count of under $0.5 \mathrm{~g} \mathrm{l}^{-1}$, often under $0.1 \mathrm{~g} \mathrm{l}^{-1}$. Many drug categories have been reported to be involved in drug-induced agranulocytosis, such as antithyroids, antiplatelets, antipsychotics and anti-inflammatory agents. $\beta$-Lactams, including cephalosporins, sulfasalazine and sulfamethoxazole/ trimethoprim and vancomycin are reported to be the most common causes of anti-infective drug-induced agranulocytosis. The median duration of $\beta$-lactam exposure before onset of acute agranulocytosis is reported in a range of 19-25 days [10]. The median time between onset of $\beta$-lactam-associated agranulocytosis and normalization of neutrophil count has been reported to be in the range of 4-12 days. Regarding our patient, ceftobiprole or acetylsalicylic acid were considered as causes of agranulocytosis and stopped, while the time interval between onset of agranulocytosis and perindopril and metoprolol treatment was rather short, and such side-effects are not described.

As acetylsalicylic acid rarely causes agranulocytosis and was tolerated well by the patient for years before mitral valve replacement and was tolerated again after normalization of neutrophil counts, we excluded acetylsalicylic acid as the offender and considered ceftobiprole as the most likely cause of agranulocytosis. The duration of ceftobiprole exposure (18 days) before agranulocytosis occurred and the time of recovery ( 8 days) are in the range of occurrence of $\beta$-lactam-associated agranulocytosis.

The pathogenesis of drug-induced agranulocytosis appears complex, as nonimmune and immune mechanisms might take place [9]. Reactive oxygen species may oxidize drugs, leading to altered drug metabolites, causing immune reactions against the neutrophil or the neutrophil precursors [11]. Host genetics or epigenetics (the interaction of drugs with gene expression) may play a role in development [12]. Drug-drug [13] and disease-drug interactions [14] have been reported.

Immunological mechanisms may play an important role in $\beta$-lactam antibiotic-associated agranulocytosis. The involvement of antibodies has been documented for penicillins and cephalosporins [15], but a T-cell-mediated pathomechanism is also possible. $\beta$-Lactam antibioticassociated agranulocytosis is often preceeded by a rash and fever, appears to be dose dependent and rarely lasts more than 10 days $[7,16,17]$. The fact that our patient showed a positive result to ceftobiprole in lymphocyte transformation testing supports the hypothesis of a T-cellmediated pathomechanism.

Our patient had a DRESS syndrome preceeding agranulocytosis. This syndrome is thought to be due to drugspecific T-cells [18] with cytotoxic potential. DRESS syndrome is often followed by reactivation of herpes viruses in the second or third week after the start of symptoms. Moreover, DRESS may be followed by antibody deficiency and autoimmune phenomena [19], and also by an intolerance or even allergy to another drug [20]. Such a flare-up reaction [21] seemed to have occurred with daptomycin treatment, where an increased eosinophila was observed. It is also quite possible that the DRESS and its associated immune alterations were important for the drug-induced haematological disease. Indeed, the reappearance of activated lymphocytes during agranulocytosis underlines the presence of a massive immune activation during this phase of the disease, and is a further argument that the side-effect is more immune mediated than toxic.

In summary, the present case report shows that prolonged ceftobiprole treatment can be associated with agranulocytosis. To our knowledge, this is the first report on ceftobiprole-associated agranulocytosis, and T-cells appear to be involved in the pathogenesis. Thus, we recommend careful monitoring of neutrophil counts in a prolonged treatment course with ceftobiprole. 


\section{Competing Interests}

There are no competing interests to declare.

Supported by SCAHT (Swiss Center of Applied Human Toxicology).

\section{REFERENCES}

1 Chaiken BH, Goldberg BI, Segal JP. Dilantin sensitivity; report of a case of hepatitis with jaundice, pyrexia and exfoliative dermatitis. N Engl J Med 1950; 242: 897-8.

2 Ben m'rad M, Leclerc-Mercier S, Blanche P, Franck N, Rozenberg F, Fulla Y, Guesmi M, Rollot F, Dehoux M, Guillevin L, Moachon L. Drug-induced hypersensitivity syndrome: clinical and biologic disease patterns in 24 patients. Medicine (Baltimore) 2009; 88: 131-40.

3 Naisbitt DJ, Farrell J, Wong G, Depta JP, Dodd CC, Hopkins JE, Gibney CA, Chadwick DW, Pichler WJ, Pirmohamed M, Park BK. Characterization of drug-specific T cells in lamotrigine hypersensitivity. J Allergy Clin Immunol 2003; 111: 1393-403.

4 Barbour A, Schmidt S, Rand KH, Derendorf H. Ceftobiprole: a novel cephalosporin with activity against Gram-positive and Gram-negative pathogens, including methicillin-resistant Staphylococcus aureus (MRSA). Int J Antimicrob Agents 2009; 34: 1-7.

5 Noel GJ, Bush K, Bagchi P, lanus J, Strauss RS. A randomized, double-blind trial comparing ceftobiprole medocaril with vancomycin plus ceftazidime for the treatment of patients with complicated skin and skin-structure infections. Clin Infect Dis 2008; 46: 647-55.

6 Janssen-Cilag AG. Zevtera ${ }^{\mathrm{TM}}$. Product monograph. In: Arzneimittelkompendium der Schweiz. Basel: Documed, 2010.

7 Olaison L, Belin L, Hogevik H, Alestig K. Incidence of beta-lactam-induced delayed hypersensitivity and neutropenia during treatment of infective endocarditis. Arch Intern Med 1999; 159:607-15.

8 Pichler WJ, Tilch J. The lymphocyte transformation test in the diagnosis of drug hypersensitivity. Allergy 2004; 59 : 809-20.

9 Tesfa D, Keisu M, Palmblad J. Idiosyncratic drug-induced agranulocytosis: possible mechanisms and management. Am J Hematol 2009; 84: 428-34.

10 Andersohn F, Konzen C, Garbe E. Systematic review: agranulocytosis induced by nonchemotherapy drugs. Ann Intern Med 2007; 146: 657-65.

11 Uetrecht JP. Reactive metabolites and agranulocytosis. Eur J Haematol Suppl 1996; 60: 83-8.

12 Kindmark A, Jawaid A, Harbron CG, Barratt BJ, Bengtsson OF, Andersson TB, Carlsson S, Cederbrant KE, Gibson NJ, Armstrong M, Lagerström-Fermér ME, Dellsén A, Brown EM,
Thornton M, Dukes C, Jenkins SC, Firth MA, Harrod GO, Pinel TH, Billing-Clason SM, Cardon LR, March RE.

Genome-wide pharmacogenetic investigation of a hepatic adverse event without clinical signs of immunopathology suggests an underlying immune pathogenesis.

Pharmacogenomics J 2008; 8: 186-95.

13 Bower M, McCall-Peat N, Ryan N, Davies L, Young AM, Gupta S, Nelson M, Gazzard B, Stebbing J. Protease inhibitors potentiate chemotherapy-induced neutropenia. Blood 2004; 104: 2943-6.

14 Coleman MD. Dapsone-mediated agranulocytosis: risks, possible mechanisms and prevention. Toxicology 2001; 162 : 53-60.

15 Lee D, Dewdney JM, Edwards RG, Neftel KA, Wälti M. Measurement of specific lgG antibody levels in serum of patients on regimes comprising high total dose beta-lactam therapy. Int Arch Allergy Appl Immunol 1986; 79: 344-8.

16 Murphy MF, Metcalfe P, Grint PC, Green AR, Knowles S, Amess JA, Waters AH. Cephalosporin-induced immune neutropenia. $\mathrm{Br}$ J Haematol 1985; 59: 9-14.

17 Peralta G, Sanchez-Santiago MB. [Beta-lactam-induced neutropenia. An old forgotten companion]. Enferm Infecc Microbiol Clin 2005; 23: 485-91.

18 Wu Y, Farrell J, Pirmohamed M, Park BK, Naisbitt DJ. Generation and characterization of antigen-specific CD4+, CD8+, and CD4+CD8+ T-cell clones from patients with carbamazepine hypersensitivity. J Allergy Clin Immunol 2007; 119: 973-81.

19 Kano $Y$, Shiohara T. The variable clinical picture of drug-induced hypersensitivity syndrome/drug rash with eosinophilia and systemic symptoms in relation to the eliciting drug. Immunol Allergy Clin North Am 2009; 29: 481-501.

20 Mennicke M, Zawodniak A, Keller M, Wilkens L, Yawalkar N, Stickel F, Keogh A, Inderbitzin D, Candinas D, Pichler WJ. Fulminant liver failure after vancomycin in a sulfasalazine-induced DRESS syndrome: fatal recurrence after liver transplantation. Am J Transplant 2009; 9: 2197-202.

21 Pichler WJ. Drug hypersenstivity reactions: classifications and relationship to T-cell activation. In: Drug Hypersensitivity, ed. Pichler WJ. Basel: Karger, 2007; 168-89.

\section{RECEIVED}

7 May 2010

\section{ACCEPTED}

17 October 2010

\section{CORRESPONDENCE}

Thomas Wendland MD, Universitätsklinik für Allgemeine Innere Medizin, Inselspital Bern, Freiburgstrasse, $\mathrm{CH}-3010$ Bern, Schweiz Tel.: +4131632 2111

Fax: +41316328885

E-mail: thomas.wendland@insel.ch 